\title{
Parallelogram-free distance-regular graphs having completely regular strongly regular subgraphs
}

\author{
Hiroshi Suzuki
}

Received: 29 December 2007 / Accepted: 19 January 2009 / Published online: 4 February 2009

(C) Springer Science+Business Media, LLC 2009

\begin{abstract}
Let $\Gamma=(X, R)$ be a distance-regular graph of diameter $d$. A parallelogram of length $i$ is a 4-tuple $x y z w$ consisting of vertices of $\Gamma$ such that $\partial(x, y)=\partial(z, w)=$ $1, \partial(x, z)=i$, and $\partial(x, w)=\partial(y, w)=\partial(y, z)=i-1$. A subset $Y$ of $X$ is said to be a completely regular code if the numbers
\end{abstract}

$$
\pi_{i, j}=\left|\Gamma_{j}(x) \cap Y\right| \quad(i, j \in\{0,1, \ldots, d\})
$$

depend only on $i=\partial(x, Y)$ and $j$. A subset $Y$ of $X$ is said to be strongly closed if

$$
\{x \mid \partial(u, x) \leq \partial(u, v), \partial(v, x)=1\} \subset Y, \text { whenever } u, v \in Y .
$$

Hamming graphs and dual polar graphs have strongly closed completely regular codes. In this paper, we study parallelogram-free distance-regular graphs having strongly closed completely regular codes. Let $\Gamma$ be a parallelogram-free distanceregular graph of diameter $d \geq 4$ such that every strongly closed subgraph of diameter two is completely regular. We show that $\Gamma$ has a strongly closed subgraph of diameter $d-1$ isomorphic to a Hamming graph or a dual polar graph. Moreover if the covering radius of the strongly closed subgraph of diameter two is $d-2, \Gamma$ itself is isomorphic to a Hamming graph or a dual polar graph. We also give an algebraic characterization of the case when the covering radius is $d-2$.

Keywords Distance-regular graph · Association scheme · Homogeneity · Completely regular code

H. Suzuki $(\bowtie)$

Department of Mathematics and Computer Science, International Christian University, Mitaka, Tokyo 181-8585, Japan

e-mail: hsuzuki@icu.ac.jp 


\section{Introduction}

The study of completely regular codes in a distance-regular graph has a long history $[3,5]$. Most of the completely regular codes studied are those with large minimum distance because of the requirements to apply the theory to error-correcting codes. Recently Brouwer et al. [2] studied a special class of completely regular codes in a $Q$-polynomial distance-regular graph satisfying extremal conditions from a different point of view. Let us call these codes extremal. These extremal codes afford induced structure of a $Q$-polynomial distance-regular graph and hence they are necessarily connected as a graph or minimum distance one. Independently, we studied the Terwilliger algebra with respect to a subset in [9]. The thin condition of the principal module of this Terwilliger algebra is equivalent to the complete regularity of the base subset. We also gave a sufficient condition, called tight, that the module generated by an end-point-zero vector is thin. In the case of the principal module, if the subset is extremal, then it is tight.

In a recent paper [10], H. Tanaka classified all extremal completely regular codes in certain classical association schemes. For example if the underlying graph is a dual polar graph, then extremal codes are strongly closed. In the literature, one also finds weak-geodesically closed used in place of strongly closed.

In this paper, we study a converse, i.e., we classify parallelogram-free distanceregular graphs having strongly closed completely regular codes. To state our results, we make a few definitions. For notation, terminology and the general theory of distance-regular graphs, we refer the reader to [1].

Let $\Gamma=(X, R)$ be a connected graph of diameter $d$ with vertex set $X$ and edge set $R$. For vertices $x$ and $y, \partial(x, y)$ denotes the distance between $x$ and $y$, i.e., the length of a shortest path connecting $x$ and $y$. More generally, for each $x \in X$ and a subset $S \subset X$ we write $\partial(x, S)=\min \{\partial(x, s) \mid s \in S\}$.

For a vertex $u \in X$ and $j \in\{0,1, \ldots, d\}$, let

$$
\Gamma_{j}(u)=\{x \in X \mid \partial(u, x)=j\} \text { and } \Gamma(u)=\Gamma_{1}(u) .
$$

A subset $Y$ of $X$ is said to be completely regular, or a completely regular code, if the following numbers

$$
\pi_{i, j}=\left|\Gamma_{j}(x) \cap Y\right| \quad(i, j \in\{0,1, \ldots, d\})
$$

depend only on $i=\partial(x, Y)$ and $j$. We write $\gamma_{i}=\pi_{i, i}$. For $Y \subset X$, the number $t(Y)=$ $\max \{\partial(x, Y) \mid x \in X\}$ is called the covering radius of $Y$, and $w(Y)=\max \{\partial(x, y) \mid$ $x, y \in Y\}$ is called the width of $Y$.

For two vertices $u$ and $v \in X$ with $\partial(u, v)=j$, let

$$
\begin{aligned}
& C(u, v)=C_{j}(u, v)=\Gamma_{j-1}(u) \cap \Gamma(v), \\
& A(u, v)=A_{j}(u, v)=\Gamma_{j}(u) \cap \Gamma(v), \text { and } \\
& B(u, v)=B_{j}(u, v)=\Gamma_{j+1}(u) \cap \Gamma(v) .
\end{aligned}
$$


A connected graph $\Gamma$ is said to be distance-regular or a distance-regular graph if the cardinalities $c_{j}=|C(u, v)|, a_{j}=|A(u, v)|$ and $b_{j}=|B(u, v)|$ depend only on $j=\partial(u, v)$ for all $j \in\{0,1, \ldots, d\}$. These numbers $c_{j}$ 's, $a_{j}$ 's and $b_{j}$ 's are called the intersection numbers of $\Gamma$.

A subset $Y$ of the vertex set $X$ is often called a code, but in this paper, it is also regarded as the induced subgraph on $Y$. A nonempty subset $Y$ of $X$ is said to be strongly closed if

$$
C(u, v) \cup A(u, v) \subset Y \text { for all } u, v \in Y .
$$

In this case $Y$ is also called a strongly closed subgraph. For two vertices $x$ and $y$, $\ll x, y \gg$ denotes the smallest strongly closed subgraph containing $x$ and $y$. Note that since the intersection of two strongly closed subgraphs is strongly closed and $\Gamma$ itself is a strongly closed subgraph containing $x$ and $y, \ll x, y \gg$ always exists.

A parallelogram of length $i$ is a 4-tuple $x y z w$ consisting of vertices of $\Gamma$ such that $\partial(x, y)=\partial(z, w)=1, \partial(x, z)=i$, and $\partial(x, w)=\partial(y, w)=\partial(y, z)=i-1$.

A parallelogram of length 2 is isomorphic to $K_{2,1,1}$. If a distance-regular graph $\Gamma$ does not have a parallelogram of length 2 , then it is said to have order $(s, t)$ for some positive integers $s$ and $t$, as every edge is contained in a maximal clique of constant size $s+1$, and every vertex is contained in exactly $t+1$ maximal cliques. In particular, the valency $k=s(t+1)$ and the neighborhood $\Gamma(x)$ of each vertex $x$ is isomorphic to a disjoint union of $t+1$ cliques of size $s$. If $c_{2}=1$, then $\Gamma$ is of order $(s, t)$ for some positive integers $s$ and $t$. If $a_{1}=0$ then $\Gamma$ is of order $(1, k-1)$.

A distance-regular graph $\Gamma=(X, R)$ of diameter $d$ is said to be a regular near polygon if it is of order $(s, t)$ for some integers $s$ and $t$, and for every maximal clique $L$ and a vertex $x \in X$ with $\partial(x, L)=i<d,\left|\Gamma_{i}(x) \cap L\right|=1$. A regular near polygon having the property that no maximal clique is contained in $\Gamma_{d}(x)$ for any $x \in X$ is called a regular near $2 d$-gon. A regular near 4 -gon is called a generalized quadrangle. A regular near polygon is often defined as an incidence structure, and in that case our regular near polygon is called the collinearity graph of a regular near polygon, or the point graph of it. See [1, Section 6.4].

If a graph does not contain parallelograms of any length, it is called parallelogram free. A regular near polygon is parallelogram free, and the parallelogram-free condition is closely related to the existence of strongly closed subgraphs. See Theorem 2.2.

Throughout this paper by strongly regular graphs we mean distance-regular graphs of diameter two, hence connected.

Now we state our main results.

Theorem 1.1 Let $\Gamma=(X, R)$ be a parallelogram-free distance-regular graph of diameter $d \geq 4$ such that $b_{1}>b_{2}$ and $a_{2} \neq 0$. Suppose every strongly closed subgraph $C$ of diameter 2 is completely regular. Then the following hold.

(i) $\Gamma$ is a regular near polygon with $c_{2}>1$, and for every pair of vertices $x, y$ at distance $d-1, \Gamma$ has a strongly closed subgraph $Y$ of diameter $d-1$ containing $x$ and $y$.

(ii) The covering radius $t(C)$ of each strongly closed subgraph $C$ of diameter 2 is at least $d-2$, and 
(a) If $t(C)=d-2$, then $\Gamma$ is isomorphic to a Hamming graph or a dual polar graph.

(b) If $t(C) \geq d-1$, then every strongly closed subgraph $Y$ of diameter $d-1$ is isomorphic to a Hamming graph or a dual polar graph.

When $q=1$ and $d \geq 4$, we can prove that $\Gamma$ itself is isomorphic to a Hamming graph without assuming that the covering radius is $d-2$ by $[6,11]$. See the last section.

We have the following characterization of the case that a strongly regular subgraph is completely regular with covering radius $d-2$.

Theorem 1.2 Let $\Gamma=(X, R)$ be a parallelogram-free distance-regular graph of or$\operatorname{der}(s, t)$ and diameter $d \geq 4$. Suppose $b_{1}>b_{2}$ and $a_{2} \neq 0$. Let $q=c_{2}-1$. Then the following are equivalent.

(i) There is a completely regular code $C$ of covering radius $d-2$ such that the induced subgraph on $C$ is strongly regular.

(ii) There is a strongly closed completely regular code $C$ of width 2 and covering radius $d-2$.

(iii) Every strongly closed subgraph of diameter 2 is completely regular with covering radius $d-2$.

(iv) Every strongly closed subgraph of diameter 2 is completely regular with covering radius $d-2$ and that it is a generalized quadrangle.

(v) $q \neq 0$ and $\Gamma$ has eigenvalues $-t-1$ and $s-t / q$.

(vi) $\Gamma$ is isomorphic to a Hamming graph or a dual polar graph.

\section{Preliminaries}

Lemma 2.1 ([1, Remark on page 86], [8, Lemma 2.6]) Let $\Gamma$ be a strongly regular graph with $a_{2} \neq 0$, and let $u$ be a vertex of $\Gamma$. Then the induced subgraph $\Delta$ on $\Gamma_{2}(u)$ is connected of diameter at most three.

Theorem 2.2 ([12, Proposition 6.7], [8, Theorem 1.1]) Let $\Gamma=(X, R)$ be $a$ distance-regular graph of diameter $d$, and let $m$ be a positive integer such that $2 \leq$ $m \leq d$. Assume that $\Gamma$ contains no parallelogram of length $i$ for any $i=2, \ldots, m+1$ and that $b_{1}>b_{2}$. In addition assume one of the following:

(i) $m=2, c_{2}>1$ and $a_{2} \neq 0$,

(ii) $c_{2}>1$ and $a_{1} \neq 0$,

(iii) $m=2$ and $c_{2}=1$,

(iv) $c_{2}=1$ and $a_{1} \neq 0$, or

(v) $c_{m+1}=1$.

Then for any vertices $x, y \in X$ with $\partial(x, y) \leq m$, the diameter of the strongly closed subgraph $\ll x, y \gg$ is $\partial(x, y)$. In particular, if $a_{2} \neq 0$, then for any vertices $x, y \in X$ with $\partial(x, y)=2$, there is a strongly closed subgraph of diameter 2 containing $x$ and $y$. 
Lemma 2.3 ([12, Lemma 6.9], [8, Lemma 4.1]) Let $\Gamma=(X, R)$ be a distanceregular graph with diameter $d \geq 3$. Suppose $\Gamma$ contains no parallelogram of any length. Let $x$ be a vertex and $Y$ a strongly closed subgraph of diameter 2. Suppose $u \in \Gamma_{i}(x) \cap Y$ and $\Gamma_{i+2}(x) \cap Y \neq \emptyset$ with $i+2 \leq d$. Then for all $y \in Y$, we have $\partial(x, y)=i+\partial(u, y)$.

\section{Terwilliger algebras and completely regular codes}

Let $\Gamma=(X, R)$ be a connected graph of diameter $d$ and $C$ a subset of $X$ with width $w=w(C)$ and covering radius $t=t(C)$. Let $C_{i}=\{x \in X \mid \partial(x, C)=i\}$ for $i \in$ $\{0,1, \ldots, t\}$.

Let $V=C^{X}=\operatorname{Span}(\hat{x} \mid x \in X)$ be a vector space over the complex number field consisting of the set of column vectors with rows indexed by the elements of $X$, and $\hat{x}$ denotes the unit vector whose $x$-entry is 1 and 0 otherwise.

For each $i=0,1, \ldots, d$, let $A_{i} \in \operatorname{Mat}_{X}(\boldsymbol{C})$ be the $i$-th adjacency matrix defined by

$$
\left(A_{i}\right)_{x, y}= \begin{cases}1 & \partial(x, y)=i \\ 0 & \text { otherwise }\end{cases}
$$

We call $A=A_{1}$ the adjacency matrix of $\Gamma$.

For $i \in\{0,1, \ldots, t\}, E_{i}^{*}=E_{i}^{*}(C) \in \operatorname{Mat}_{X}(C)$ are defined as follows.

$$
\left(E_{i}^{*}\right)_{x, y}= \begin{cases}1 & \text { if } x=y \text { and } x \in C_{i}, \\ 0 & \text { otherwise. }\end{cases}
$$

The matrix $E_{i}^{*}$ induces the projection onto the subspace $E_{i}^{*} V=\operatorname{Span}\left(\hat{x} \mid x \in C_{i}\right)$.

Definition 3.1 The Terwilliger algebra $\mathcal{T}=\mathcal{T}(C)$ of a connected graph $\Gamma=(X, R)$ associated with a subset $C$ of $X$ is a matrix subalgebra over $C$ of $\operatorname{Mat}_{X}(\boldsymbol{C})$ generated by $A$ together with $E_{0}^{*}, E_{1}^{*}, \ldots, E_{t}^{*}$, where $t=t(C)$. A $\mathcal{T}$-module $W$ is a $\mathcal{T}$-invariant linear subspace of $V$. A nonzero $\mathcal{T}$-module $W$ is said to be irreducible if $W$ does not contain proper nonzero $\mathcal{T}$-modules. An irreducible $\mathcal{T}$-module $W$ is said to be thin if $\operatorname{dim} E_{i}^{*} W \leq 1$ for every $i=0,1, \ldots, t$.

Definition 3.2 Let $\Gamma=(X, R)$ be a connected graph, and $C$ a nonempty subset of $X$. Let $\mathbf{1}_{C}=\sum_{x \in C} \hat{x} \in V=C^{X}$. Then $C$ is said to be a completely regular code if $\mathcal{T}(C) \mathbf{1}_{C}$ is a thin irreducible $\mathcal{T}(C)$-module.

Note that if $\Gamma$ is a distance-regular graph, the definition of complete regularity in the introduction coincides with the one given above. The proof is straightforward. See [9, Proposition 7.2] and [5].

Let $\Gamma=(X, R)$ be a connected graph. Then it is immediate that $\Gamma$ is distanceregular if and only if it is regular and every singleton $\{x\}$ with $x \in X$ is completely regular. It is not difficult to show that if $\Gamma$ is distance-regular of diameter $d$, then every edge $\{x, y\}$ with $x, y \in X$ is completely regular if and only if $a_{1}=a_{2}=\cdots=$ $a_{d-1}=0$, i.e., $\Gamma$ is almost bipartite or bipartite. 
Thin Irreducible Modules. Let $\Gamma=(X, R)$ be a distance-regular graph of valency $k$ and diameter $d$. Let $A_{i}$ be the $i$-th adjacency matrix and $A=A_{1}$. Then there is a polynomial $v_{i}(\lambda) \in C[\lambda]$ of degree exactly $i$ such that $v_{i}(A)=A_{i}$. Let $k_{i}=v_{i}(k)$. Then $k_{i}=\left|\Gamma_{i}(x)\right|$ for every $x \in X$. Let $\theta_{0}>\theta_{1}>\cdots>\theta_{d}$ be distinct eigenvalues of $A$ and let $E_{0}, E_{1}, \ldots, E_{d}$ be the primitive idempotents of $\boldsymbol{C}[A]$ corresponding to each of the distinct eigenvalues. Then each column of $E_{i}$ is an eigenvector of the same eigenvalue $\theta_{i}$ of $A$, and $A E_{i}=\theta_{i} E_{i}$. Let $m\left(\theta_{i}\right)=\operatorname{tr}\left(E_{i}\right)$. Then $m\left(\theta_{i}\right)$ is the multiplicity of $\theta_{i}$ as an eigenvalue of $A$. Set $\Theta=\left\{\theta_{0}, \theta_{1}, \ldots, \theta_{d}\right\}$.

Let $C$ be a nonempty subset of $X$ and $\mathcal{T}=\mathcal{T}(C)$. We consider an irreducible $\mathcal{T}$-module $W$ such that $E_{0}^{*} W \neq 0$, which is called a module of endpoint 0 .

We review some facts proved in [9].

Let $\boldsymbol{v}=E_{0}^{*} \boldsymbol{v}$ be a nonzero vector. Set

$$
\rho \boldsymbol{v}(\lambda)=\frac{1}{|X|} \sum_{i=0}^{d} \frac{\overline{{ }^{t} \boldsymbol{v}} A_{i} \boldsymbol{v}}{\|\boldsymbol{v}\|^{2}} \frac{v_{i}(\lambda)}{k_{i}} \in \boldsymbol{R}[\lambda] .
$$

The following is called the inner distribution of the vector $\boldsymbol{v}$.

$$
a(\boldsymbol{v})=\left(\frac{\overline{{ }^{\bar{v}}} A_{0} \boldsymbol{v}}{\|\boldsymbol{v}\|^{2}}, \ldots, \frac{\overline{{ }^{\bar{v}}} A_{i} \boldsymbol{v}}{\|\boldsymbol{v}\|^{2}}, \ldots, \frac{\overline{{ }^{t} \boldsymbol{v}} A_{d} \boldsymbol{v}}{\|\boldsymbol{v}\|^{2}}\right) .
$$

By definition, if $w=w(C)$ is the width of $C$, then the degree of $\rho \boldsymbol{v}(\lambda)$ is at most $w$. On the other hand by direct computation we have

$$
\frac{\left\|E_{i} \boldsymbol{v}\right\|^{2}}{\|\boldsymbol{v}\|^{2}}=\rho \boldsymbol{v}\left(\theta_{i}\right) m\left(\theta_{i}\right) .
$$

Since $\boldsymbol{C}[A] \boldsymbol{v}=\operatorname{Span}\left(E_{0} \boldsymbol{v}, E_{1} \boldsymbol{v}, \ldots, E_{d} \boldsymbol{v}\right)$, we have

$$
\operatorname{dim} C[A] \boldsymbol{v} \geq d+1-(\# \text { of roots of } \rho \boldsymbol{v}(\lambda) \text { in } \Theta) \geq d+1-w(C) .
$$

Set $r=r(\boldsymbol{v})=\operatorname{dim} \boldsymbol{C}[A] \boldsymbol{v}-1$. The number $r(\boldsymbol{v})$ is called the dual degree of $\boldsymbol{v}$. If $\mathbf{1}_{C}$ is the characteristic vector of $C$, we write $r(C)$ for $r\left(\mathbf{1}_{C}\right)$ and call the dual degree of $C$. Now we have the following.

Theorem 3.1 ([9, Theorem 1.1]) Let $\Gamma=(X, R)$ be a distance-regular graph of diameter $d$, and $C$ a nonempty subset of $X$. Let $E_{0}^{*}=E_{0}^{*}(C)$ and $\boldsymbol{v}=E_{0}^{*} \boldsymbol{v}$ a nonzero vector. Then the following hold.

(i) $\operatorname{dim} \boldsymbol{C}[A] \boldsymbol{v}+w(C) \geq d+1$.

(ii) If $\operatorname{dim} C[A] \boldsymbol{v}+w(C)=d+1$, then $\mathcal{T}(C) v$ is a thin irreducible $\mathcal{T}(C)$-module.

A nonzero vector $v \in E_{0}^{*} V$ satisfying the condition in Theorem 3.1 (ii) is called a tight vector. When $E_{0}^{*} V$ is spanned by tight vectors, we call $C$ a tight code.

The case that $\boldsymbol{v}$ is the characteristic vector $\mathbf{1}_{C}$ of $C$ is also studied in [2]. See also [4]. 
Corollary 3.2 ([2, Theorem 1$])$ Let $\Gamma=(X, R)$ be a distance-regular graph of diameter $d$, and $C$ a nonempty subset of $X$ with dual degree $r=r(C)$. If $r+w(C)=d$, then $C$ is a completely regular code. Moreover, we have $t=r$ in this case.

Note that the condition in the corollary can be checked if we have $a\left(\mathbf{1}_{C}\right)$ together with the set of eigenvalues of $A$. In the literature, the inner distribution $a\left(\mathbf{1}_{C}\right)$ is also called the inner distribution of the code $C$ and denoted $a(C)$.

\section{Completely regular subgraphs}

Proposition 4.1 Let $\Gamma=(X, R)$ be a distance-regular graph of valency $k$ and diameter $d$. Let $C$ be a subset of $X$ contained in a proper strongly closed subgraph $Y$ of $\Gamma$. In addition assume that $\left|\Gamma_{i}(z) \cap C\right|$ depends only on $i$ whenever $\partial(z, C)=1$. Then $C$ is strongly closed.

Proof First note that the maximal valency of $Y$ is not $k$. Suppose not, and let $m$ be the diameter of $Y$. Then $c_{m}+a_{m}=k$ and $b_{m}=0$. This implies $m=d$ and $Y$ is not regular. This contradicts Theorem 1.1 in [7].

Let $x, y \in C$ such that $\partial(x, y)=\ell$. Since the maximal valency of $Y$ is less than $k$, there is a vertex $u \in X \backslash Y$ adjacent to $x$. Let $v \in C$. Since $C \subset Y$ and $Y$ is strongly closed $\partial(u, v)=\partial(x, v)+1$. Let $z \in \Gamma(x)$ such that $\partial(z, y) \leq \ell$. We claim that $z \in C$. Suppose not. Then $\partial(z, C)=1$ and the following hold.

$$
\sum_{v \in C} \partial(x, v)+|C|=\sum_{v \in C} \partial(u, v)=\sum_{v \in C} \partial(z, v) .
$$

Since $\partial(z, v) \leq \partial(x, v)+1$. The above holds only if $\partial(z, v)=\partial(x, v)+1$ holds for all $v \in C$. Since $y \in C$ and $\partial(z, y) \leq \ell=\partial(x, y)$, this is absurd. Thus we proved the claim. Hence $C$ is strongly closed.

An induced subgraph on $Y$ of a graph $\Gamma=(X, R)$ is called weakly closed if the distance in the subgraph is equal to the distance in $\Gamma$.

Corollary 4.2 Let $\Gamma=(X, R)$ be a distance-regular graph of diameter $d$. Let $C$ be a weakly closed distance-regular subgraph in $\Gamma$ of diameter $\ell$, and $u, v \in C$ with $\partial(u, v)=\ell$. In addition assume that $\left|\Gamma_{i}(z) \cap C\right|$ depends only on $i$ whenever $\partial(z, C)=1$. If both $u$ and $v$ are contained in a proper strongly closed subgraph $Y$ of $\Gamma$, then $C \subset Y$ and $C$ is strongly closed.

Proof By Proposition 4.1, it suffices to show that $C \subset Y$. Since $C$ is connected for each $w \in C$, there is a path $u=u_{0} \sim u_{1} \sim \ldots \sim u_{m}=w$ in $C$. Since the diameter of $C$ is $\ell, C$ is weakly closed and $Y$ is strongly closed, $C \cap \Gamma(u) \subset Y$ and $C \cap \Gamma(v) \subset Y$. Since $C$ is distance-regular and weakly closed, there is a vertex $v_{1} \in(\Gamma(v) \cup\{v\}) \cap C$ such that $\partial\left(u_{1}, v_{1}\right)=\ell$. Since $v_{1} \in Y$, we can proceed by induction to show $w \in Y$. 
Lemma 4.3 Let $\Gamma=(X, R)$ be a distance-regular graph of diameter $d$. Let $1 \leq m \leq$ $d-1$ be an integer. Suppose for $u, v \in X$ with $\partial(u, v)=m$, there is a strongly closed subgraph $C$ of diameter $m$ containing $u$ and $v$ and $C$ is completely regular. Then the parameters $\pi_{i, j}$ of $C$ are determined by $m$ and the parameters of $\Gamma$.

Proof Since $C$ is strongly closed in $\Gamma$, the parameters of $C$ and hence the inner distribution of $C$ is determined by the parameters of $\Gamma$ and $m$. Now the assertion follows from [9, Corollary 10.3].

\section{Completely regular strongly regular subgraphs}

In this section, we study parallelogram-free distance-regular graphs having completely regular strongly regular subgraphs. The goal is to establish the following result.

Theorem 5.1 Let $\Gamma=(X, R)$ be a parallelogram-free distance-regular graph of diameter $d \geq 4$ such that $b_{1}>b_{2}$ and $a_{2} \neq 0$. Suppose every strongly closed subgraph $C$ of diameter 2 is completely regular. Let $c_{2}=q+1$. Then $\Gamma$ is a regular near polygon, $q \geq 1$ and $c_{i}=\left[\begin{array}{l}i \\ 1\end{array}\right]_{q}$ for $i \in\{1,2, \ldots, d-1\}$. Moreover if the covering radius of $C$ is $d-2$, then $c_{d}=\left[\begin{array}{l}d \\ 1\end{array}\right]_{q}$ and $\Gamma$ is a regular near $2 d$-gon.

We first remark that under the hypothesis of Theorem 5.1, for two vertices $x, y$ with $\partial(x, y)=2$, there is a strongly closed subgraph $\ll x, y \gg$ of diameter 2 containing $x$ and $y$ by Theorem 2.2 .

Hypothesis 5.1 Let $\Gamma=(X, R)$ be a parallelogram-free distance-regular graph of diameter $d \geq 4$ such that $b_{1}>b_{2}$ and $a_{2} \neq 0$. Every strongly closed subgraph $C$ of diameter 2 is completely regular.

Let $s=a_{1}+1$ and $t=b_{1} / s$. Then $\Gamma$ in Hypothesis 5.1 is of order $(s, t)$.

Lemma 5.2 Under Hypothesis 5.1, for every $i \leq d-2$ and $u \in X$ with $\partial(u, C)=i$, $\gamma_{i}=\gamma_{i}(u)=\left|C \cap \Gamma_{i}(u)\right|=1, \alpha_{i}=\alpha_{i}(u)=\left|C \cap \Gamma_{i+1}(u)\right|=\kappa=a_{2}+c_{2}$. In particular, the covering radius of $C$ is at least $d-2$ and the parameters $\gamma_{i}$ and $\alpha_{i}$ of $C$ as a completely regular code do not depend on the choice of strongly closed subgraphs of diameter 2 up to $i \leq d-2$.

Proof Let $x, y \in C$ with $\partial(x, y)=2$. Since $i \leq d-2$, there is a vertex $u \in \Gamma_{i}(x) \cap$ $\Gamma_{i+2}(y)$. Then by Lemma 2.3, we have the desired conclusion. Since $C$ is completely regular, this is the case for all $u \in X$ with $\partial(u, C)=i$.

Lemma 5.3 Under Hypothesis 5.1, C is a generalized quadrangle. In particular $c_{2}>1$. 
Proof Since $\Gamma$ is parallelogram free and $C$ is strongly closed, $C$ is of order $(s, \tau)$ for some integer $\tau$. Let $u \in C$. Suppose that there are adjacent vertices $v, w \in \Gamma_{2}(u) \cap C$ such that $A(v, w) \subset \Gamma_{2}(u)$. Let $x \in B(u, w)$. Since $C$ is strongly closed, $\partial(v, x)=2$. Let $C^{\prime}=\ll v, x \gg$. Since $\gamma_{2}=1$ and $v, w \in C \cap \Gamma_{2}(u), \partial\left(u, C^{\prime}\right)=1$. Let $\{y\}=$ $\Gamma(u) \cap C^{\prime}$. Then $v, w$ and all vertices in $C^{\prime} \cap \Gamma_{2}(u)$ are in $\Gamma(y)$, which is absurd as $\{v, w\} \cup A(v, w)$ is a maximal clique. Hence $C$ is a generalized quadrangle.

Lemma 5.4 Under Hypothesis 5.1, $\Gamma$ is a regular near polygon. Moreover if the covering radius of $C$ is $d-2$, then $\Gamma$ is a regular near $2 d$-gon.

Proof Let $L$ be a maximal clique and $\partial(u, L)=i \leq d-1$ for some vertex $u$. We will show that $\left|\Gamma_{i}(u) \cap L\right|=1$. We may assume that $i \geq 2$ as $L$ is a maximal clique. By way of contradiction assume that two vertices $v$ and $w$ are in $\Gamma_{i}(u) \cap L$.

First assume that $\Gamma_{i+1}(u) \cap L=\emptyset$. Let $x \in C(u, v)$. Then $\partial(x, w)=2$. Let $C=\ll$ $x, w \gg$. Then either $\partial(u, C)=i-1$ or $\partial(u, C)=i-2$. The first case does not occur as otherwise $\partial(x, w)=1$ by Lemma 5.2. Suppose $\partial(u, C)=i-2$. By Lemma 5.3, we have a contradiction as we assumed that $\Gamma_{i+1}(u) \cap L=\emptyset$. This part also proves that if the covering radius of $C$ is $d-2$, there is no maximal clique $L$ such that $\partial(u, L)=d$.

Next assume that $\Gamma_{i+1}(u) \cap L \neq \emptyset$. Let $x \in \Gamma_{i+1}(u) \cap L$ and $y \in C(u, v)$. Then $\partial(x, y)=2$. Let $C=\ll x, y \gg$. Since $v, w \in C$, this contradicts Lemma 5.2.

Lemma 5.5 Let $q=c_{2}-1$. Under Hypothesis 5.1 the following hold.

$$
c_{i+1}-1=\left(c_{2}-1\right) c_{i}, \text { and } c_{i+1}=1+q+\cdots+q^{i}=\left[\begin{array}{c}
i+1 \\
1
\end{array}\right]_{q} \text { for all } i \leq d-2
$$

Moreover, if every strongly closed subgraph $C$ of diameter 2 is of covering radius $d-2$, then (1) holds for $i=d-1$ as well.

Proof Let $u, v, w \in X$ with $\partial(u, v)=i+1 \leq d$ and $w \in C(u, v)$. We count the number of pairs in the following set.

$$
N=\{(x, y) \mid x \in C(u, w), y \in C(x, v) \backslash\{w\}\} .
$$

First there are $c_{i}$ choices of $x$ and then for each $x \in C(u, w)$, there are $c_{2}-1$ choices of $y$. Hence we have $|N|=\left(c_{2}-1\right) c_{i}$.

Next let $y \in C(u, v) \backslash\{w\}$. Since $\Gamma$ is a regular near polygon by Lemma 5.4, $\partial(y, w)=2$. Let $Y$ be the strongly closed subgraph of diameter 2, containing $y$ and $w$. Since $\{y, w\} \subset \Gamma_{i}(u)$ and $v \in Y \cap \Gamma_{i+1}(u), \partial(u, Y)=i-1$ if $i \leq d-2$ or $i=d-1$ and every strongly closed subgraph $C$ of diameter 2 is of covering radius $d-2$. By Lemma 5.2, there exists a vertex $x$ such that $\Gamma_{i-1}(u) \cap Y=\{x\}$ and that $y, w \in \Gamma(x)$. Therefore $x$ is the unique vertex in $C(y, w) \cap \Gamma_{i-1}(u)$. Hence $(x, y) \in N$ and $|N|=$ $c_{i+1}-1$.

Since $q=c_{2}-1$ and $c_{i+1}=q c_{i}+1$, we have the formula for $c_{i+1}$ by induction. 
Proof of Theorem 5.1 Since $C$ is a generalized quadrangle with $a_{2} \neq 0$ by Lemma 5.3, $c_{2} \geq 2$ and $q \geq 1$. Now we have the assertions by Lemma 5.4 and Lemma 5.5.

Proof of Theorem 1.1 By Theorem $5.1 c_{2}>1$ and $\Gamma$ is a regular near polygon. Since $a_{2} \neq 0, a_{1} \neq 0$. Hence for every pair of vertices $x, y$ at distance $d-1, \Gamma$ has a strongly closed subgraph $Y$ of diameter $d-1$ containing $x$ and $y$ by Theorem 2.2. Let $Y$ be a strongly closed subgraph of diameter $d-1$ in $\Gamma$. Then $Y$ is a regular near $2(d-1)$ gon with $c_{i}=\left[\begin{array}{l}i \\ 1\end{array}\right]_{q}$. Hence it is with classical parameters $\left(d-1, q, 0, a_{1}+1\right)$. Now $Y$ is isomorphic to a Hamming graph or a dual polar graph if $d \geq 4$ by Theorem 9.4.4 in [1]. The covering radius of $C$ is at least $d-2$ by Lemma 5.2 and the result for the case the covering radius is $d-2$ follows similarly using the characterization in [1, Theorem 9.4.4].

\section{Tight completely regular codes of small width}

In this section, we consider the case that a subset $C$ of small width $w \leq 2$ becomes a completely regular code with smallest covering radius $d-w$ or $\mathbf{1}_{C}$ is tight that satisfies the condition in Corollary 3.2.

Lemma 6.1 Let $C$ be a subset of a distance-regular graph $\Gamma=(X, R)$ of diameter $d \geq 2$. Let $\boldsymbol{v}$ be a non-zero vector such that $\operatorname{supp}(\boldsymbol{v}) \subset C$. Let

$$
\rho_{\boldsymbol{v}}(\lambda)=\frac{1}{|X|} \sum_{i=0}^{d} \eta_{i} \frac{v_{i}(\lambda)}{k_{i}} \in \boldsymbol{R}[\lambda] \text {, where } \eta_{i}=\eta_{i}(\boldsymbol{v})=\frac{\overline{{ }^{t} \boldsymbol{v}} A_{i} \boldsymbol{v}}{\|\boldsymbol{v}\|^{2}} .
$$

Then the following hold.

(i) If $w(C)=1$, then

$$
\rho \boldsymbol{v}(\lambda)=\frac{1}{|X| b_{0}}\left(b_{0}+\eta_{1} \lambda\right)
$$

(ii) If $w(C)=2$, then

$$
\rho \boldsymbol{v}(\lambda)=\frac{1}{|X| b_{0} b_{1}}\left(b_{0}\left(b_{1}-\eta_{2}\right)+\left(\eta_{1} b_{1}-\eta_{2} a_{1}\right) \lambda+\eta_{2} \lambda^{2}\right) .
$$

Proof Since

$$
v_{0}(\lambda)=1, v_{1}(\lambda)=\lambda, \text { and } c_{2} \cdot v_{2}(\lambda)=\lambda^{2}-a_{1} \lambda-b_{0},
$$

the formulas above follow by direct computation using the fact that $\eta_{0}=1$ and $\eta_{i}=0$ for all $i>w(C)$.

Corollary 6.2 Let $C$ be a subset of a distance-regular graph $\Gamma=(X, R)$ of order $(s, t)$ of diameter $d \geq 2$. Let $\mathbf{1}_{C}$ be the characteristic vector of $C$. Then the following hold. 
(i) Suppose $C$ is a maximal clique of size $s+1$. Then

$$
\rho_{\mathbf{1}_{C}}(\lambda)=\frac{1}{|X|(t+1)}(t+1+\lambda) .
$$

(ii) Suppose $C$ is strongly regular and strongly closed. In addition assume that $c_{2}+$ $a_{2}=(q+1) s$ with $q=c_{2}-1$, i.e., $C$ is a generalized quadrangle. Then

$$
\rho_{\mathbf{1}_{C}}(\lambda)=\frac{1}{|X|(t+1) t}(q \lambda+t-q s)(\lambda+t+1) .
$$

Proof (i) is immediate. For (ii), $\eta_{0}=1, \eta_{1}=(q+1) s$ and $\eta_{2}=q s^{2}$. Hence the formula is immediate.

Proposition 6.3 Let $\Gamma=(X, R)$ be a distance-regular graph of order $(s, t)$ of diameter $d \geq 3$. Suppose $C$ is a strongly closed generalized quadrangle in $\Gamma$. Then the following are equivalent.

(i) $\Gamma$ has eigenvalues $-t-1$ and $s-t / q$, where $q=c_{2}-1$.

(ii) $C$ is completely regular with covering radius $d-2$.

Moreover if (i), (ii) hold, then every maximal clique $C_{1}$ is completely regular with covering radius $d-1$.

Proof This is a direct consequence of Theorem 3.1, Corollary 3.2 and Corollary 6.2.

Proof of Theorem 1.2 (iv) $\Rightarrow$ (iii) $\Rightarrow$ (ii) $\Rightarrow$ (i) is clear, and (vi) $\Rightarrow(v)$ is well-known. See $[1$, p. 261, p. 276].

(i) $\Rightarrow$ (ii): Since the diameter of $C$ is two, it is weakly closed. Hence by Corollary 4.2, $C$ is strongly closed.

(ii) $\Rightarrow$ (iii): Let $\rho(\lambda)=\rho_{\mathbf{1}_{C}}(\lambda)$. Since $\rho(\lambda)$ is determined by $\kappa_{1}=|\Gamma(x) \cap C|=c_{2}+a_{2}$ and $\kappa_{2}=\left|\Gamma_{2}(x) \cap C\right|=\left(c_{2}+a_{2}\right)\left(c_{2}-a_{2}-s\right) / c_{2}, \rho$ does not depend on the choice of strongly closed subgraph. Moreover by (ii), two distinct eigenvalues of $\Gamma$ are the roots of $\rho$. Therefore, every strongly closed subgraph of diameter 2 is completely regular with covering radius $d-2$.

(iii) $\Rightarrow$ (iv): We need to show that the induced subgraph on $C$ is a generalized quadrangle. This follows from Lemma 5.3.

(v) $\Rightarrow$ (iv): Since $\Gamma$ has an eigenvalue $-t-1$, every maximal clique of size $s+1$ is a completely regular code with covering radius $d-1$. Let $C$ be a strongly closed subgraph of diameter 2 . Then every maximal clique of size $s+1$ contained in $C$ is completely regular with covering radius 1 . Since $C$ is of order $(s, \tau)$ with a suitable choice of an integer $\tau, C$ is a generalized quadrangle. In particular $q \neq 0$ as $a_{2} \neq 0$. Note that if $Y$ is a maximal clique and $x \in C \backslash Y,|\Gamma(x) \cap Y|=1$ as $Y$ is maximal. 
Hence $\rho_{C}$ is as in Corollary 6.2 and $\rho_{C}$ has two eigenvalues $-t-1$ and $s-t / q$ as roots. Therefore, $C$ is completely regular with covering radius $d-2$.

(iv) $\Rightarrow$ (vi): This is a direct consequence of Proposition 6.3 and Theorem 1.1.

\section{Remarks}

For the case $q=1$, the following two propositions cover most of our results. We only sketch their proofs.

Theorem 7.1 Let $\Gamma$ be a parallelogram-free distance-regular graph of order $(s, t)$ with $c_{2}=2, a_{2}=2(s-1)$ and $c_{3}=3$ with $s>1$. If the diameter $d \geq 3$, then $\Gamma$ is isomorphic to the Hamming graph $H(d, s+1)$.

Proof We proceed by induction on $d$. If $d=3$, then by [6, Corollary], $\Gamma$ is isomorphic to $H(3, s+1)$. Note that we do not need the assumption $s \neq 3$ as $\Gamma$ is parallelogram free. Suppose the assertion holds for $d-1$. By Theorem 2.2, there is a strongly closed subgraph $\Delta$ of diameter $d-1$ in $\Gamma$. By induction hypothesis, $\Delta$ is isomorphic to $H(d-1, s+1)$ with $d \geq 4$. Now by [6, Theorem 1], there is a $(d-1)$-error correcting completely regular code of covering radius $d$ in a $H(n, s+1)$ with $s+1 \geq 3$. These are uniformly packed codes classified by $H$. van Tilborg [11] and the only possibility for $\Gamma$ is $H(d, s+1)$.

Corollary 7.2 Let $\Gamma$ be a parallelogram-free distance-regular graph of order $(s, t)$, diameter $d \geq 4$ with $c_{2}=2$. Suppose $\Gamma$ contains a strongly regular (vertex induced) subgraph with parameters $(\kappa, \lambda, \mu)$. If $\kappa \neq \mu$ and $\pi_{i, j}=\left|\Gamma_{j}(x) \cap C\right|$ depends only on $i=\partial(x, C)$ and $j$ whenever $(i, j)=(1,1),(1,2)$ or $(2,2)$. Then $\Gamma$ is isomorphic to the Hamming graph $H(d, s+1)$.

Proof By our assumption, $c_{2}>1$ and $a_{2} \neq 0$. By Theorem 2.2, for each pair of distance two there is a strongly closed subgraph of diameter two containing the pair. Hence by Corollary 4.2, $C$ is strongly closed. Now by Lemma $2.3, \pi_{1,1}=1$, $\pi_{1,2}=\kappa=c_{2}+a_{2}$ and $\pi_{2,2}=1$. Hence by the proof of Lemma 5.3, $C$ is a generalized quadrangle and $a_{2}=2(s-1)$. By mimicking the proof of Lemma 5.5, $c_{3}=3$. We are now ready to apply Theorem 7.1 to conclude that $\Gamma$ is isomorphic to $H(d, s+1)$.

The consideration of the case $q=1$ above suggests us to classify distance-regular graphs of order $(s, t)$ of diameter $d \geq 4$ with the following parameters:

$$
c_{i}=1+q+\cdots+q^{i-1}, a_{i}=c_{i}(s-1) \text { for all } i \in\{1,2, \ldots, d-1\}
$$

with $q \geq 2$ and $s>1$.

The results in this paper also suggest problems to characterize distance-regular graphs by a given completely regular subgraph. Since $\Gamma_{d}(x)$ is always completely regular, this problem is connected to the problem to characterize distance-regular 
graphs by the structure of $\Gamma_{d}(x)$. We close this paper by giving a possible improvement of the result of this paper.

Replace the hypothesis 'parallelogram-free' in Theorem 5.1 and Corollary 5.1 by the following:

$\Gamma$ is of order $(s, t)$ and every maximal clique is completely regular.

\section{References}

1. Brouwer, A.E., Cohen, A.M., Neumaier, A.: Distance-Regular Graphs. Springer, Berlin (1989)

2. Brouwer, A.E., Godsil, C.D., Koolen, J.H., Martin, W.J.: Width and dual width of subsets in polynomial association schemes. J. Comb. Theory A 102, 255-271 (2003)

3. Delsarte, P.: An algebraic approach to the association schemes of coding theory. Philips Research Reports Supplements 1973, No.10

4. Hosoya, R., Suzuki, H.: Tight distance-regular graphs with respect to subsets of width two. Eur. J. Comb. 28, 61-74 (2007)

5. Neumaier, A.: Completely regular codes. Discrete Math. 106-107, 353-360 (1992)

6. Nomura, K.: Distance-regular graphs of Hamming type. J. Comb. Theory B 50, 160-167 (1990)

7. Suzuki, H.: On strongly closed subgraphs of highly regular graphs. Eur. J. Comb. 16, 197-220 (1995)

8. Suzuki, H.: Strongly closed subgraphs of a distance-regular graph with geometric girth five. Kyushu J. Math. 50, 371-384 (1996)

9. Suzuki, H.: The Terwilliger algebra associated with a set of vertices in a distance-regular graph. J. Algebr. Comb. 22, 5-38 (2005)

10. Tanaka, H.: Classification of subsets with minimal width and dual width in Grassmann, bilinear forms and dual polar graphs. J. Comb. Theory A 113,903-910 (2006)

11. van Tilborg, H.C.A.: Uniformly packed codes. Ph.D. thesis, Eindhoven (1976). http:// alexandria.tue.nl/extra1/PRF2B/7602641.pdf

12. Weng, C.: Weak-geodesically closed subgraphs in distance-regular graphs. Graphs Comb. 14, 275304 (1998) 